\title{
Correspondence
}

\section{Epidural anaesthesia and pulmonary hypertension}

To the Editor:

I read with interest the article in the January 1983 issue describing the use of thoracic epidural anaesthesia in a patient with congenital heart disease and pulmonary hypertension. ${ }^{1}$

The cardiac catheterization data as presented do not supply definite evidence of partial anomalous pulmonary venous retum (PAPVR). Indirect evidence of PAPVR can be provided by demonstrating a rise in blood oxygen saturation in the innominate vein, superior vena cava, inferior vena cava, or right atrium, depending upon the site of insertion of the anomalous vein. However, when this rise is evident in the right atrium, as in this case, the only information that it supplies is that there is a left to right shunt at that level, and the possible causes of this are several. The most accurate diagnosis comes from injection of contrast medium into the pulmonary artery and examining the pulmonary venous return of each lung. ${ }^{2}$ This enables an accurate statement regarding the pulmonary venous return to be made. The author unfortunately failed to substantiate the diagnosis with such angiographic information.

The comments made regarding pulmonary sympathetic innervation and lack of a decrease in pulmonary artery pressure after sympathetic denervation by cpidural blockade also deserve consideration.

Dr. Maliampati states that the fall in pulmonary artery pressure seen in this patient was hardly significant in spite of what was most likely complete block of the pulmonary sympathetic innervation. He then attributed this to the decreased vascular smooth musculature and decreased innervation of the pulmonary vasculature in comparison with the systemic vasculature.

As was stated, increased pulmonary flow and the secondarily increased pulmonary artery pressure, "... triggers anatomic changes in small pulmonary arteries in the form of medial hypertrophy, intimal hyperplasia and fibrosis. These changes per se increase resistance to pulmonary blood flow and exacerbate the increased pulmonary arterial pressure. The net effect is a vicious cycle of deterioration, lead to cor pulmonale." This is in essence correct; however, it has been shown in infants with congenital heart disease that abnormal growth and development of the pulmonary vascular bed is present on a congenital rather than an acquired basis and the effects of elevated pulmonary blood flow and pulmonary artery pressure are superimposed upon an already abnormal pulmonary vasculature. ${ }^{3}$

In postmortem studies the axial arteries are dilated at the hilum of the lung but narrow peripherally, as was suggested by the chest $x$-ray in the patient. Microscopically the peripheral pulmonary arteries were seen to be small in size and few in number. The muscular component of the pulmonary vessels are seen to extend more peripherally into the normally nonmuscular peripheral pulmonary arteries. The normal perinatal regression of the thickened pulmonary musculature does not occur and additional medial hypertrophy has been demonstrated and may increase the medial thickness to greater than two times normal., ${ }^{4,5}$ If the pulmonary blood flow and pulmonary artery pressure are not decreased to normal values, the development of fasciculi of longitudinal muscle both internal and external to the hypertrophied media occurs. At the same time as this is occurring cellular intimal proliferation is seen to occur which produces first narrowing and then complete obliteration of the pulmonary vessel lumen. ${ }^{6}$ This tends to "fix" pulmonary artery resistance and make it minimally or nonresponsive to therapeutic intervention.

The current literature therefore notes an increase in the musculature component of pulmonary arteries in individuals with congenital heart disease and increased pulmonary blood fow and pulmonary arterial pressure as compared with the lesser musculature of normals. With this increased musculature it might be expected that sympathetic effects would 
be amplified and that sympathetic block would cause a marked decrease in pulmonary artery pressure unless sympathetic innervation is minimal, as Dr. Mallampati pointed out. More likely, however, is that the patient had developed pulmonary vascular changes sufficient to preclude significant effect from sympathetic blockade. The cardiac catheterization data reveal pulmanary artery pressure greater than one-half systemic with a markedly elevated pulmonary vascular resistance. The pulmonary artery pressure was seen to be minimally responsive to the inhalation of 100 per cent oxygen or the administration of sodium nitroprusside and without calculation of the pulmonary vascular resistance it is difficult to state whether or not the pulmonary vascular resistance was affected at all. It is not surprising therefore that no effect was seen from sympathetic denervation.

The data Dr. Mallampati presents does not substantiate any of the comments regarding lack of effect of sympathetic blockade. Such data should be best obtained from an individual with normal pulmonary vasculature.

Frederick A. Burrows MD FRCP(C)

Section of Pediatric Cardiovascular Anesthesia Arkansas Children's Hospital

Little Rock, Arkansas, 72201

\section{REFERENCES}

1 Mallampati SR. Low thoracic epidural anaesthesia for elective cholecystectomy in a patient with congenital heart disease and pulmonary hypertension. Can Anaesth Soc J 1983; 30: 72-6

2 Rowe $R D$. Anomalies of venous retum. In: heart disease in infancy and childhood, Ed Keith JD, Rowe RD, Vlad P. New York: Macmillan, 1978 , pp. 554-89.

3 Rabinovifch $M$, Reid LM. Quantitacive structural analysis of the pulmonary vascular bed in congenital heart defects in pediatric cardiovascular disease, Ed Engle MA. Philadelphia: F.A. Davis, 1981, pp. 149-69.

4 Hislop A, Haworth SG, Reid LM. Quantitative structural analysis of pulmonary vesse] in isolated ventricular septal defects in infancy. Br Heart $J$ 1975; 37 : 1014-21.

5 Haworth SG, Sauer U, Buhlmeyer K, Reid LM. The development of the pulmonary circulation in ven- tricular septal defect: A quantitative structural study. Am J Cardiol 1977; 40: 781-88.

6 Heath $D$, Edwards $J E$. The pathology of hypertensive pulmonary vascular disease. Circulation 1958; 18: 533-47.

\section{$R E P L Y$}

$I$ would like to thank Dr. Burrows for his comments. Please allow me to make it clear that the observations published pertain to the perioperative care of an unusual case. As has been pointed out in the discussion, there were no precedents in the amaesthesia literature for comparative purposes.

The diagnosis was established by an experienced team including a clinician, an invasive cardiologist and a diagnostic radiologist. The patient had undergone both right and lefi heart catheterization, left ventriculogram. and echocardiography. Manual injections of conirast medium into vessels encountered during right heart catheterization revealed ane anomalous pulmanary vein from the midportion of the right lung draining into the right atrium and another one from the left lung draining into the left innominate vein. An atrial septal defect or ventricular septal defect was not demonstrable by echocardiography or catheler search. Injection of large doses of contrast medium for pulmonary angiography, which Dr. Burrows refers to, is not without complications in patients with elevated pulmonary vascular resistance. Acute hypotension and bradyarrhythmias are common. This was therefore deemed an undesirable risk to the patient considering that the information obtained would not have altered the course of management. Most of Dr. Burrows' remaining conments refer to the pulmonary vascular disease in infants and children secondary to congenital heart defects. His comments on the pathologic changes in pulmonary vessels are informative.

As he points out, there is more smooth muscle in pulmonary arteries in individuals with congenital heart disease and increased pulmonary blood flow. But that pathologic finding does not tell us anything about the response of the smooth muscle to sympathetic stimulation or denervation, as compared with this response in individuals with normal pulmonary vasculature. Dr. Burrows suggests that such data should be best obtained from patients with normul pulmonary vasculature. To the best of my knowledge, no one has conducted a study to ascertain the effect of pulmonary sympathetic blockade an pulmonary haemodynamic sin individuals with normal pulmonary vasculature.

S. Rao Mallampati MD FaCA Department of Anesthesia Brigham and Women's Hospital, Boston, Massachusetts, 02115 